\title{
Mixed Tumor in Deep Lobe and Versatility of Acellular Dermal Matrix
}

\author{
Jin Hwan Byun, \\ Jung Soo Lim, \\ Hye Kyung Lee \\ Department of Plastic and Reconstructive \\ Surgery, Nowon Eulji Medical Center, Eulji \\ University School of Medicine, Seoul, Korea
}

No potential conflict of interest relevant to this article was reported.

\begin{abstract}
Frey's syndrome and infra-auricular depressed deformities are the ones of the most common complications that can occur after total parotidectomy. We report 1 case of pleomorphic adenoma occurred in the deep lobe that obtained good results from using acellular dermal matrix (ADM) after total parotidectomy. A 24-year-old man visited the hospital with oval shape mass in right mandibular angle which of $4 \mathrm{~cm}$ in size was found in the deep lobe of right parotid gland from Magnetic resonance imaging scanning and a pleomorphic adenoma was suspected. A total parotidectomy was performed while preserving the facial nerve. The material known as ADM were placed in the depressed part from where the mass was removed, and the site was sutured. The surgery site was healed well without any complications such as Frey's syndrome or infra-auricular depressed deformities. The pathological result was confirmed as pleomorphic adenoma. In addition to these advantages, it does not have little potential of deformation by the gravity after the surgery, and there is no restraint on circulation, which makes fabrication free and each deformation into various shapes can be described as another advantage of the reconstruction using the ADM.
\end{abstract}

Keywords: Acellular dermis / Pleomorphic adenoma / Parotid neoplasm / Sweating, gustatory

\section{INTRODUCTION}

Because Frey's syndrome or infra-auricular depressed deformities occurs well after total parotidectomy, the studies on the effective prevention measures for it have been actively conducted. The author obtained good results from using acellular dermal matrix (ADM) after total parotidectomy in order to prevent such complications as 1 case of pleomorphic adenoma occurred in the deep lobe and we report it with the literature review.

\section{CASE REPORT}

A 24-year-old man visited the hospital with oval shape mass in

\section{Correspondence: Hye Kyung Lee}

Department of Plastic and Reconstructive Surgery, Nowon Eulji Medical Center, Eulji University School of Medicine, Hangeulbisuk-gil 68, Nowon-gu, Seoul 01830, Korea

E-mail: hklee240@eulji.ac.kr

Received August 6, 2016 / Revised May 31, 2017 / Accepted May 31, 2017 right mandibular angle, which had grown slowly from 3 years before the visit to the hospital as main complaint. There were no particular apparent traumas and no other medical history other than asthma. A soft mass of $5 \times 4 \mathrm{~cm}$ in size on physical examination was palpated, there was no presence of facial nerve weakness, and he was complaining that he felt as if the face of the affected side, was turned to ipsilateral direction (Fig. 1).

A well-defined lobulated mass of $4 \mathrm{~cm}$ in size was found in the deep lobe of right parotid gland from Magnetic resonance imaging (MRI) scanning and a pleomorphic adenoma was suspected (Fig. 2). We planned a total parotidectomy and decided to use the ADM to correct for the predicted depression. The mass was located at deep lobe as shown in the MRI images and a total parotidectomy was performed. 5 branches of facial nerve were identified during the surgery and all of them was preserved. The material known as ADM of $4 \mathrm{~cm} \times 7 \mathrm{~cm}$ in size, $3.4 \mathrm{~mm}$ thickness (Flex HD, Ethicon Co. LLC., Somerville, New Jersey and Cincinnati, 

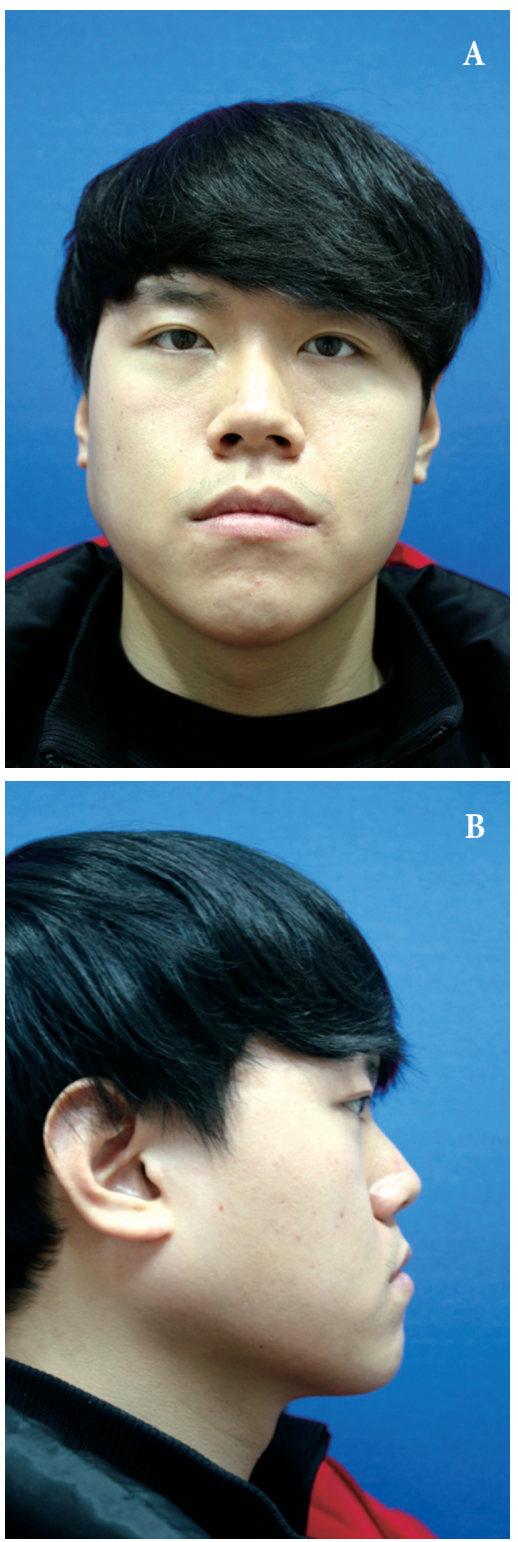

Fig. 1. A $5 \mathrm{~cm} \times 4 \mathrm{~cm}$ oval shape soft mass was palpated as motionless. Fluctuation or inflammation sign were not accompanied and such as pain, facial weakness and trismus were not observed. (A) Frontal view. (B) Profile view.

$\mathrm{OH}$, USA) were adequately trimmed for the depressed part from where the mass was removed. The part between the cheek flap and nerve were augmented centering the depressed site after the mass was removed. After the closed draining tubes were inserted over and under the ADM, the site was sutured (Figs. 3, 4).

Drains were drained by $27 \mathrm{~mL} /$ day a day after surgery in bloody character, gradually became serosanguineous, and de-

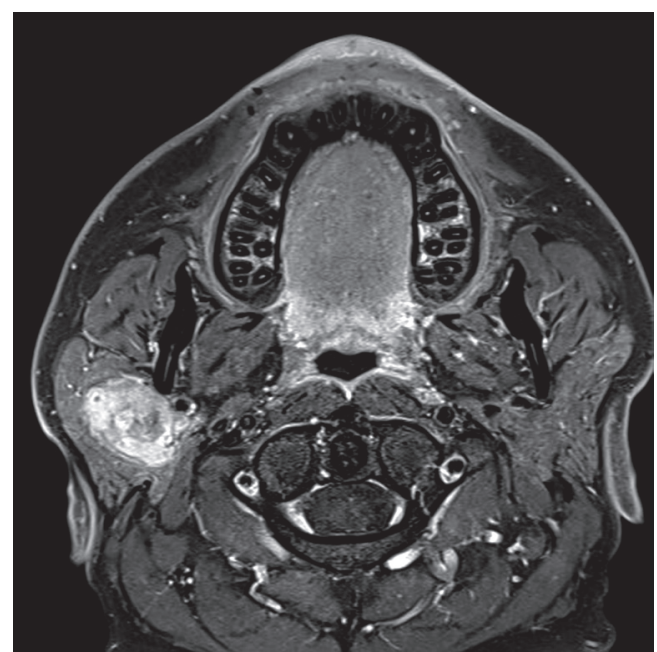

Fig. 2. It is a $4 \mathrm{~cm}$, well-defined lobulated mass in deep lobe of right parotid gland, and is located at postero-medial aspect of right retromandibular vein.

creased in volume and drained to less than $10 \mathrm{~mL} /$ day 3 days after surgery, but were removed somewhat later. We removed superficial one and cut deep one to allow drainage naturally 5 days after surgery, and removed deep one 7 days after surgery. The surgery site was healed well without any complications such as seroma or infection, and mild weakness of the depressor of the lower lip was observed (House Brackmann Grade II). This symptom was completely resolved at 4 months after the surgery. Such as Frey's syndrome were not observed, there was minimal depression and the facial symmetry was well maintained, as the patient satisfaction was high (Fig. 5). The pathological result was confirmed as pleomorphic adenoma.

\section{DISCUSSION}

Pleomorphic adenoma is a most common type of parotid tumor, and usually benign, and slow growing. But it has been known for rarely malignant degeneration [1]. Likewise this case, the parotid tumor occurred in deep lobe is far rare compared to the superficial lobe, applicable to frequency of $5 \%$ of the total parotid tumor [2].

One of the most important complications of parotid surgery for pleomorphic adenoma is recurrent disease [3]. The most important causes of recurrence are incomplete tumor excision and spillage. These are associated with histologic findings for the tu- 


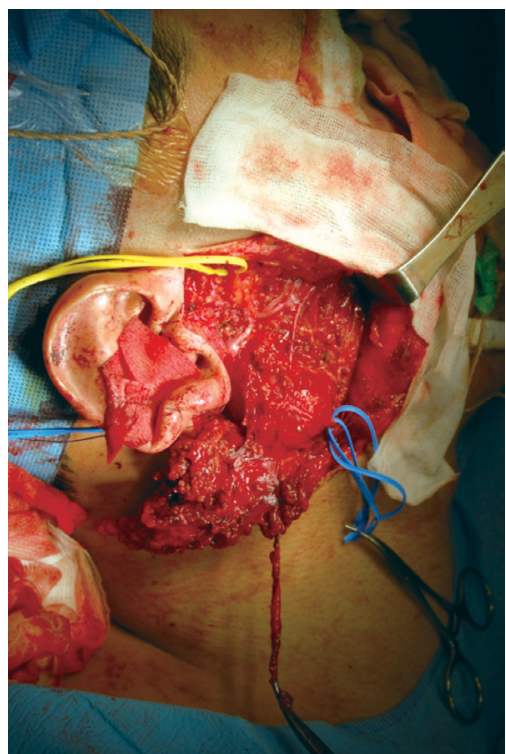

Fig. 3. Superficial lobectomy was performed ensuring 5 branches of facial nerve were well preserved facial nerve, and they were confirmed by the nerve stimulator (NIM-Response 2.0, Medtronic Inc., Jacksonville, FL, USA). The mass is seen under the running of this nerve.

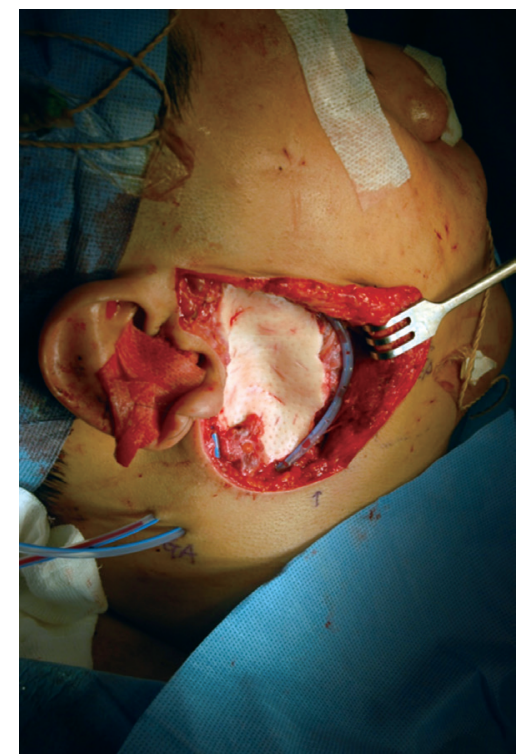

Fig. 4. An acellular dermal matrix of $4 \mathrm{~cm} \times 7 \mathrm{~cm}$ in size, $3.4 \mathrm{~mm}$ in thickness was trimmed adequately to the defect size and curvature, and its edge was cut in zig-zag shape. Several slits were made and inserted between the nerve and the cheek flap.
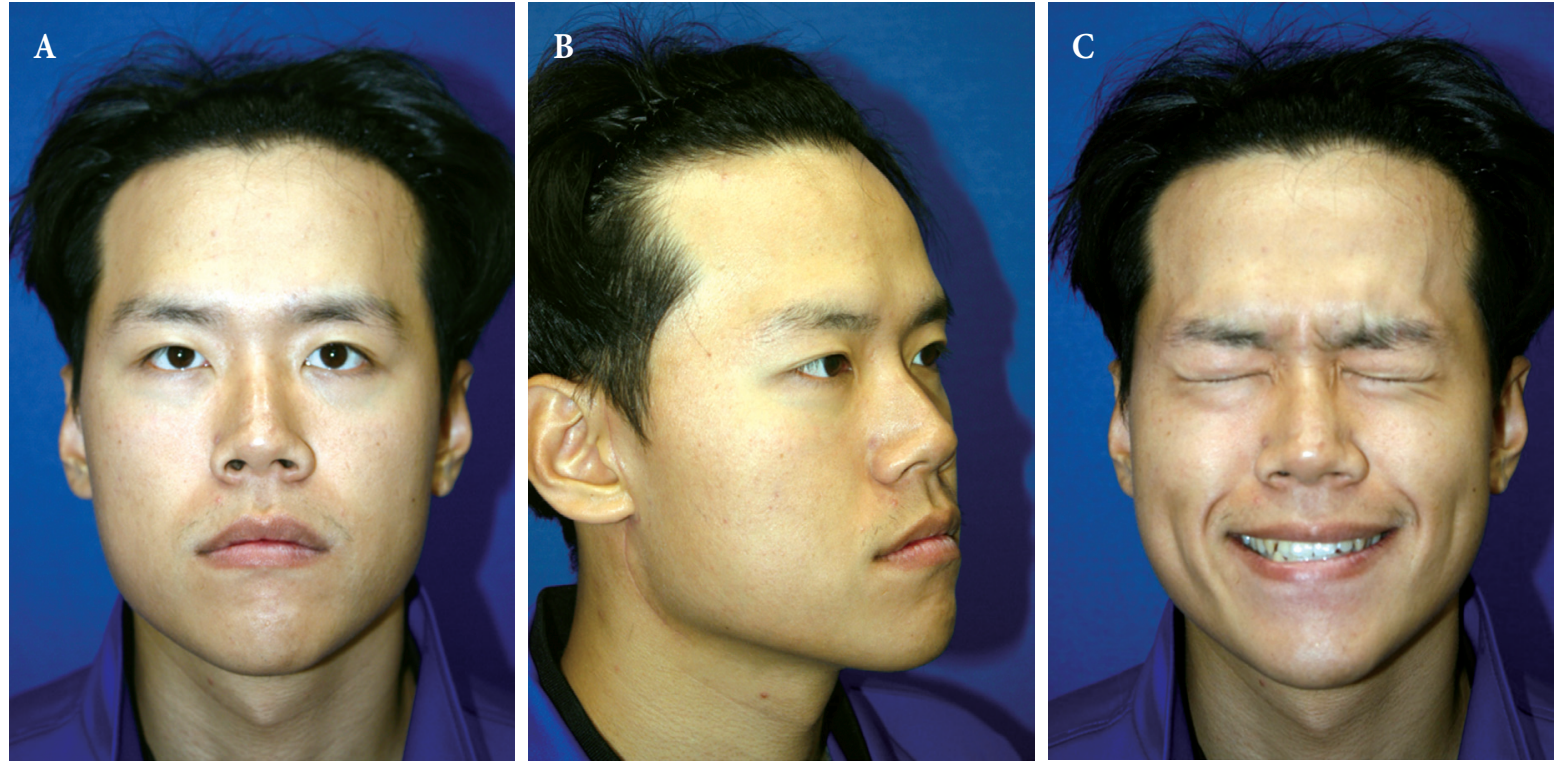

Fig. 5. Photographs at 4 months after surgery. (A) Frontal view. (B) Profile view. The patient did not complain about gustatory sweating and there was minimal depression. (C) When conducted the physical examination, the function of frontalis and depressor anguli oris muscle was symmetric, and measured as House Brackmann Grade I.

mor (multicentric nature, focal absence of the capsule, and the presence of pseudopods). Therefore, radical resection of the tumor is difficult. Furthermore, it is difficult to distinguish the facial nerve from scar tissue when performing reoperation for recurrent pleomorphic adenoma because of exposure during the first operation [4]. As the importance of removing the cuff surround the tumor was emphasized for several decades, superficial parotidectomy or total parotidectomy was successfully used to treat pleo- 
morphic adenoma, and an average recurrence rate declined to below $2 \%[5]$.

Malignant and deep parotid tumors are indications for total parotidectomy [6,7]. Features predictive of malignant change include tumors which are greater than $4 \mathrm{~cm}$ and/or demonstrate fixity, facial nerve involvement, palpable cervical nodes, and deep lobe involvement [5]. In addition, a significant risk factor for recurrence is younger age [8]. In our case, total parotidectomy was performed because the patient was 24 years old and the mass was $4 \mathrm{~cm}$ and was located in the deep lobe.

Frey's syndrome and infra-auricular depressed deformities are the ones of the most common complications that can occur after total parotidectomy. For the prevention of above-mentioned two complications, a number of scholars have been trying in diversities, including Sternocleidomastoid muscle flap, temporoparietal fascia flap, the superficial musculoaponeurotic system flap, the free or vascularized dermal fat graft, and other mesh materials such as Ethisorb (Ethicon Inc., Somerville, NJ, USA) or polytetrafluoroethylene (Gore-Tex; W. L. Gore and Associates, Flagstaff, AZ, USA) [6,9].

In particular, in case of temporoparietal fascia flap, it has relatively low donor site morbidity, compared to the above-listed other surgical methods, and its effectiveness are recognized in terms that it is enough to fill the large size of defect after total parotidectomy. However, in terms that the scar remains longer than ADM, and in terms that that there is a potential of alopecia due to its locational feature, and in terms that it requires longer operating time. The disadvantage of free or vascularized grafts of fat, dermal fat is that they are easily absorbed, which result in recurrence of the original facial contour deformity. And they require additional donor sites and leave a scar $[6,10,11]$. Other mesh materials had the shortcoming of the greater risk of rejection than ADM $[12,13]$. In addition, incidence of extrusion was rather high in polytetrafluoroethylene than ADM. In case of Ethisorb, it is absorbed over time due to its resorbable properties $[9,13]$.

ADM is derived from allograft skin, with the cellular constituents of the epithelium and dermis removed by a series of treatments. Since only type IV collagen was preserved, there is no immune response during implantation. Therefore, the possibility of rejection is low [12]. In addition to this advantage, it does not have little potential of deformation by the gravity after the surgery, and there is no restraint on circulation, which makes fabrication free and each deformation into various shapes can be described as another advantage of the reconstruction using the ADM. Therefore, ADM-utilized reconstruction was performed in this case.

Although there had been cases reported for the use of ADM in other countries to the best our knowledge, there has been no report on application to Korean. But in this case, we experienced satisfactory results without any complications such as Frey's syndrome or infra-auricular depressed deformities. Therefore, we want to add this case to the literatures related to performance of reconstruction by using other existing $\mathrm{ADM}$.

\section{REFERENCES}

1. Mulla O, Agada F, Dawson D, Sood S. Deep lobe parotid pleomorphic adenoma presenting as obstructive sleep apnoea. BMJ Case Rep 2013; 2013:pii: bcr2013008655.

2. Carr RJ, Bowerman JE. A review of tumours of the deep lobe of the parotid salivary gland. Br J Oral Maxillofac Surg 1986;24:155-68.

3. McGurk M, Thomas BL, Renehan AG. Extracapsular dissection for clinically benign parotid lumps: reduced morbidity without oncological compromise. Br J Cancer 2003;89:1610-3.

4. Zbaren P, Vander Poorten V, Witt RL, Woolgar JA, Shaha AR, Triantafyllou A, et al. Pleomorphic adenoma of the parotid: formal parotidectomy or limited surgery? Am J Surg 2013;205:109-18.

5. McGurk M, Cascarini L. Controversies in the management of salivary gland disease. 2nd ed. Oxford: Oxford University Press; 2013.

6. Luo W, Zheng X, Chen L, Jing W, Tang W, Long J, et al. The use of human acellular dermal matrix in the prevention of infra-auricular depressed deformities and Frey's syndrome following total parotidectomy. Oral Surg Oral Med Oral Pathol Oral Radiol 2012;114:e9-13.

7. To VS, Chan JY, Tsang RK, Wei WI. Review of salivary gland neoplasms. ISRN Otolaryngol 2012;2012:872982.

8. Abu-Ghanem Y, Mizrachi A, Popovtzer A, Abu-Ghanem N, Feinmesser R. Recurrent pleomorphic adenoma of the parotid gland: institutional experience and review of the literature. J Surg Oncol 2016; 114:714-8.

9. Dulguerov P, Quinodoz D, Cosendai G, Piletta P, Marchal F, Lehmann W. Prevention of Frey syndrome during parotidectomy. Arch Otolaryngol Head Neck Surg 1999;125:833-9.

10. Dai XM, Liu H, Li YS, Ji SG, Qin SH, Liu L. Prevention of Frey syndrome with temporal fascia flap in parotidectomy. Ann Plast Surg 2015;75:610-4.

11. Livesey SA, Herndon DN, Hollyoak MA, Atkinson YH, Nag A. 
Transplanted acellular allograft dermal matrix: potential as a template for the reconstruction of viable dermis. Transplantation 1995;60:1-9.

12. Ye WM, Zhu HG, Zheng JW, Wang XD, Zhao W, Zhong LP, et al. Use of allogenic acellular dermal matrix in prevention of Frey's syndrome after parotidectomy. Br J Oral Maxillofac Surg 2008;46:649-52.

13. Sinha UK, Saadat D, Doherty CM, Rice DH. Use of AlloDerm implant to prevent Frey syndrome after parotidectomy. Arch Facial Plast Surg 2003;5:109-12. 